\title{
Acoustic Purcell Effect for Enhanced Emission
}

\author{
Maryam Landi, ${ }^{1}$ Jiajun Zhao, ${ }^{2,3, \dagger}$ Wayne E. Prather, ${ }^{1}$ Ying Wu, ${ }^{2, *}$ and Likun Zhang ${ }^{1, *}$ \\ ${ }^{1}$ National Center for Physical Acoustics and Department of Physics and Astronomy, University of Mississippi, \\ University, Mississippi 38677, USA \\ ${ }^{2}$ King Abdullah University of Science and Technology (KAUST), Division of Computer, \\ Electrical and Mathematical Sciences and Engineering, Thuwal 23955-6900, Saudi Arabia \\ ${ }^{3}$ GOWell International LLC, Houston, Texas 77041, USA
}

(Received 25 August 2017; published 13 March 2018)

\begin{abstract}
We observe that our experimentally measured emission power enhancement of a speaker inside a previously proposed metacavity agrees with our numerically calculated enhancement of the density of states (DOS) of the source-cavity system. We interpret the agreement by formulating a relation between the emitted sound power and the acoustic DOS. The formulation is an analog to Fermi's golden rule in quantum emission. The formulation complements the radiation impedance theory in traditional acoustics for describing sound emission. Our study bridges the gap between acoustic DOS and the acoustic Purcell effect for sound emission enhancement.
\end{abstract}

DOI: 10.1103/PhysRevLett.120.114301

In a seminal work in 1946, Purcell predicted that an atom in a wavelength-size resonant cavity can radiate much faster than in free space [1]. It was referred to as the Purcell effect for the modification of the spontaneous emission rate of a quantum source by changing its surrounding environment via changing the density of states (DOS) in the quantum system (the number of modes per unit frequency range and per unit volume) [Fig. 1(a)]. This causal relation was elucidated by Fermi's golden rule (FGR) that implies the linear dependence of the atom's emission rate on DOS [2]. The physics and applications were widely explored in quantum systems [3-7] and recently in electromagnetic systems [8-13]. In this Letter, we develop the analog to FGR in sound emission enhancement by modifying the acoustic source's environment [Fig. 1(b)].

Emission enhancement of a sound source by environment changes has been achieved traditionally by employing coupling horns to loudspeaker drivers [14], employing various enclosure designs [15], or, more recently, by acoustic resonant metamaterial structures [16-18]. The emission enhancement is particularly important in lowfrequency emission since efficient emissions are highly demanded in audio acoustics. The high refractive index in recently designed metamaterial structures by coiled up (zigzag) space configurations [19-22] enables the enhancement at low frequencies by resonances, as recently developed for enhanced emission of a monopole source by Fabry-Perot resonances [17] and for a multipole source of arbitrary orders by degenerate Mie resonances [18]. The low-frequency resonances for enhanced emission were proved by these designs which effectively change the source's emission environment and may be hence regarded as the Purcell effect in acoustics.
In traditional acoustics, enhanced emission (or acoustic Purcell effect, per se) has been generally interpreted by acoustic resonances [16-18] and radiation impedance $[14,15]$. The fundamental questions remain: How the resonant enhancement of sound emission can be understood by the DOS of sound waves? Whether there exists an acoustic analogue of the FGR for characterizing the enhancement in terms of the acoustic DOS?

In this Letter, we develop a frame describing the emission enhancement of a sound source by the acoustic analog of FGR and gain insight into the emission by the acoustic DOS. We identified that the emission enhancement results from enhanced DOS of the sound system, which is specified by the ratio of the DOS in the presence of the enclosure and that in free space. We analytically derived and experimentally verified the acoustic analog of FGR, emitted sound power $\propto$ acoustic DOS, and revealed the physics that the emission enhancement results from the DOS enhancement.

Experimental and numerical data of emission enhancement.-First, we obtain reliable data of enhanced emission by using a previously proposed subwavelength enclosure

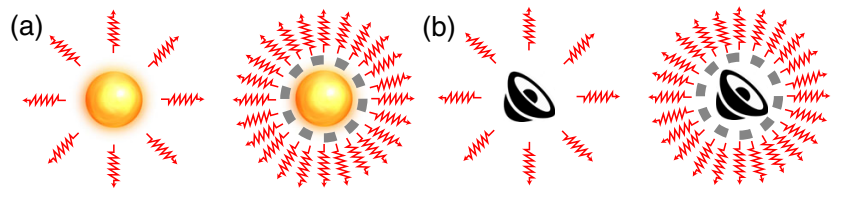

FIG. 1. Analog of resonant enhancement of emission of (a) an atom and (b) a speaker by the surrounding cavity. An acoustic analog of Fermi's golden rule that relates the emission to density of states is herein developed to characterize the sound emission enhancement. 

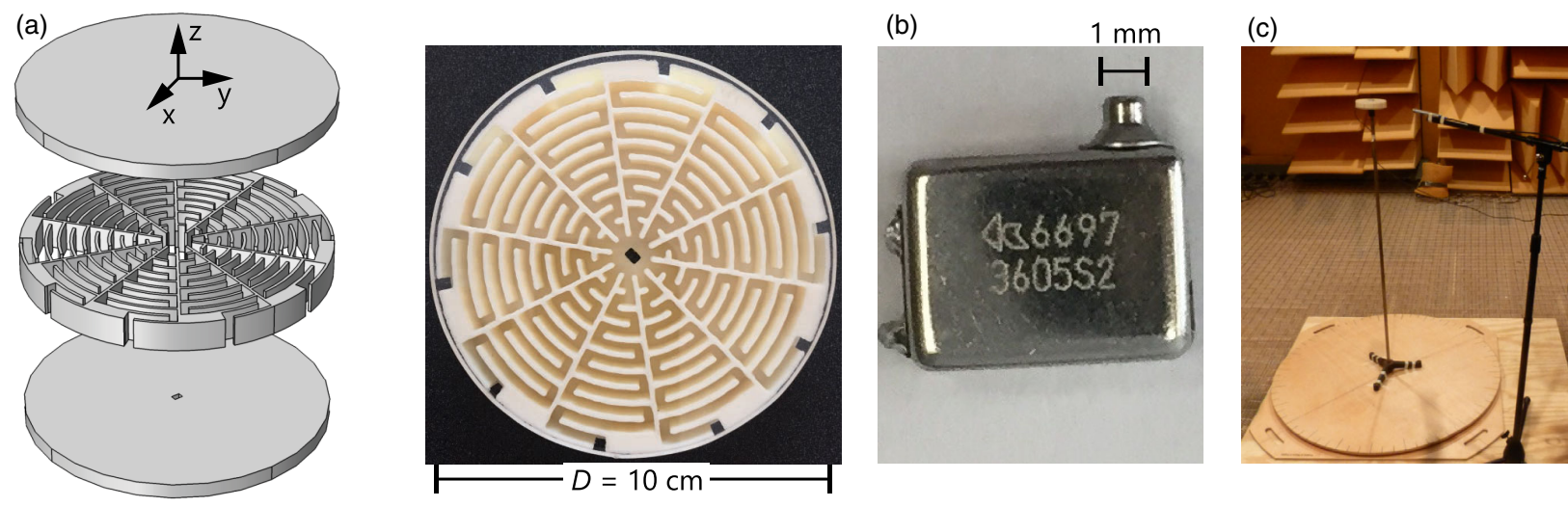

FIG. 2. Experimental setup. (a) Schematic (left) and photo (right) of acoustical cavity for resonant emission enhancement, consisting of 10 zigzag channels sandwiched between two caps, where the hole centered in the lower cap is to fit speaker tube. (b) A balanced armature speaker used as the monopole source with a 1-mm-diameter sound tube. (c) Measurements conducted in an anechoic chamber by using a microphone to pick up sound emitted by the source mounted on a turntable.

that supports Mie resonances for emission enhancement [18]. The structure is made by 3D printing out of acrylonitrile butadiene styrene plastic [Fig. 2(a)]. The coilingspace (zigzag) configuration of the channels (width $\times$ height $\times$ length $=3 \times 10 \times 170 \mathrm{~mm}^{3}$ starting from an inner radius of $5 \mathrm{~mm}$ ) elongates sound paths, resulting in a low effective radial sound speed (i.e., high refractive index [17-22]), consequently shifting the resonant frequencies down to attain low-frequency emission enhancement [18].

We employed a balanced armature speaker (Knowles, Model ID CI-26697-000, $7.2 \times 9.5 \times 4.1 \mathrm{~mm}^{3}$ ) as the monopole source [Fig. 2(b)], with the speaker mouth (1 $\mathrm{mm}$ diameter) embedded and sealed into a hole $\left(3 \times 2 \mathrm{~mm}^{2}\right)$ at the center of the structure [cf. in Fig. 2(a)]. The small size of this speaker and its efficient response at our required low frequencies make it a legitimate point source for our measurements.

Measurements were conducted by placing the enclosed speaker on a stand $1 \mathrm{~m}$ above the ground in the middle of an anechoic chamber (located at the National Center for Physical Acoustics, University of Mississippi) [Fig. 2(c)]. An Audiomatica CLIO FW-11 fire wire audio measurement system and QC Model 5 power amplifier switching and testing box were used to drive the speaker and record sound signals. In both the absence and presence of the enclosure, an impulse signal was generated and the response of the speaker was measured by a $1 / 2^{\prime \prime}$ microphone (Breuel \& Kjaer type-4191) connected to a preamplifier (Nexus microphone conditioner Type 2690-A).

Figure 3(a) displays the free-field pressure response (no enclosure) as well as the pressure response of the enclosed speaker. With the presence of the structure, enhancement appears at a full range of frequencies up to $700 \mathrm{~Hz}$ with a resonance peak of $23 \mathrm{~dB}$ (or 200 times in sound power) around $485 \mathrm{~Hz}$ (which depends on the compactness of the coiled sound path in the structure). This frequency for Mie resonance corresponds to a wavelength of $\lambda=70 \mathrm{~cm}$, confirming a large enhancement at the subwavelength region.

We show in Fig. 3(b), the enhancement peak measured as a function of distance from the center of the structure along $x$ and $z$ axes, respectively. A noticeable difference is apparent at the distances very close to the surface of the structure, where the SPL along the $z$ axis is suppressed by the caps of the structure which block the sound field.
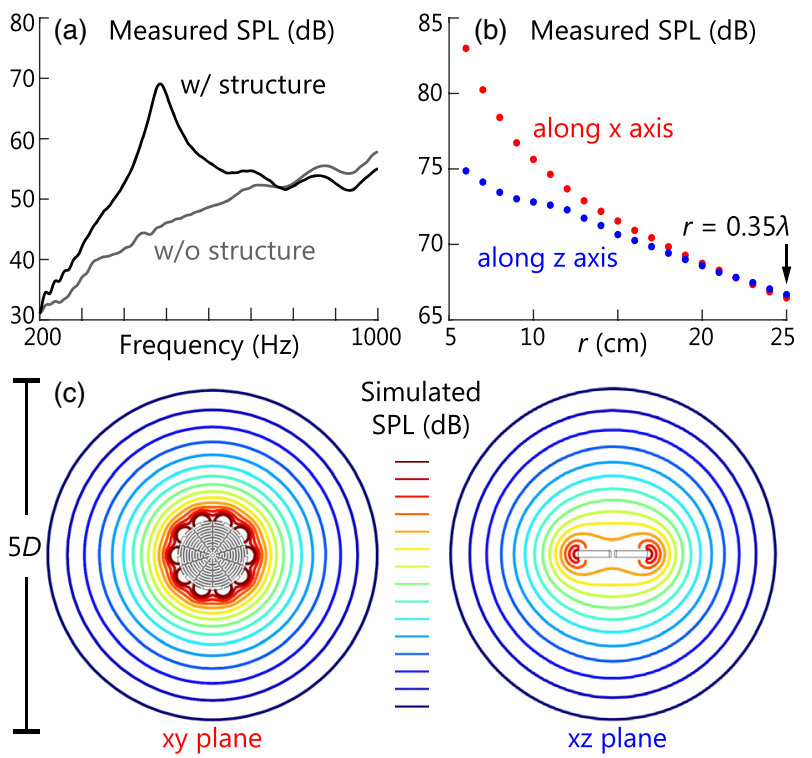

FIG. 3. Measurements and simulations. (a) Sound pressure level (SPL) with and without the cavity (measured at $15 \mathrm{~cm}$ away from the source) shows $23 \mathrm{~dB}$ enhancement at $485 \mathrm{~Hz}$, corresponding to $D=0.14 \lambda$ ( $\lambda$ is the wavelength). (b) SPL peak at $485 \mathrm{~Hz}$ measured along the $x$ and $z$ directions at a subwavelength source-to-hydrophone distance $r$. (c) SPL in $x y$ and $x z$ planes for $485 \mathrm{~Hz}$ sound by 3D simulations (where the contour lines have an incremental of $1.14 \mathrm{~dB}$ and minimum-tomaximum range of $16 \mathrm{~dB}$ ). 
However, the measurements along $x$ and $z$ axes are well converged at $r<0.35 \lambda$ ( $k r<1$ with $k$ the wave number), revealing an omnidirectional enhancement at a subwavelength environment in the three dimensional (3D) space.

The emitted sound fields in 3D space are further illustrated by results shown in Fig. 3(c) from 3D simulations with losses included in the channels of the structure [18]. The simulation was conducted with COMSOL based on a finite element method. We used the thermoacoustic module to model the losses in the channels of the structure $[18,23]$. The source was simulated with a constant source strength, radiating sound in a 3D environment [24]. The structure was treated as rigid because of a stark contrast of acoustic impedance between the material and air.

We now obtain the emission enhancement, from both simulations and measurements, characterized by the magnitude of the power enhancement and termed as the acoustic Purcell factor (APF). The measured APF was given by the squared ratio of pressure measured with and without the structure. The measurements were repeated at different angles in the $x y$ and $x z$ planes with an increment of $18^{\circ}$ azimuthal angle and with a constant source-tomicrophone distance of $15 \mathrm{~cm}$. The simulated APF was calculated from integrating sound energy flux over a spherical surface $(r=15 \mathrm{~cm})$ enclosing the structure. The APF obtained from measured sound pressure and from simulations, as a function of $D / \lambda$, are compared in Figs. 4(a) and 4(b). The measurements confirm the model prediction over the examined frequency. The good agreement between the measured and simulated results proves the reliability of our data of emission enhancing rate.

Sound emission enhancement and acoustic density of states.-Sound source's emission is usually explained in terms of acoustic radiation impedance. The radiation impedance (denoted by $Z$ ) is the ratio of sound pressure on the surface of a source to the velocity of the source [25]. The radiation efficiency is normally characterized by the ratio of resistance $\operatorname{Re}(Z)$, real part of $Z$, to the absolute value of the impedance and indicates how much energy is radiated to far field compared to the energy stored in the near field for a given source and frequency [14]. Shown in Fig. 4(c) is the radiation efficiency $\operatorname{Re}(Z) /|Z|$ calculated from simulated acoustic pressure and normal velocity on the source surface, for both cases with and without the structure. Without the structure, the efficiency $\operatorname{Re}(Z) /|Z|$ is in general low and increases monotonically with frequency. With the presence of the structure, the overall efficiency $\operatorname{Re}(Z) /|Z|$ is improved and we observe a $100 \%$ efficiency occurs at the emission enhancement maximum [cf. Figs. 4(a) and 4(b)], corresponding to resonances that lead to the radiation reactance $\operatorname{Im}(Z)=0$.

Herein we theoretically and numerically examine the DOS of the acoustic emission system. Acoustic DOS is a classical counterpart to the quantum DOS and is likewise defined as the number of modes per unit frequency range
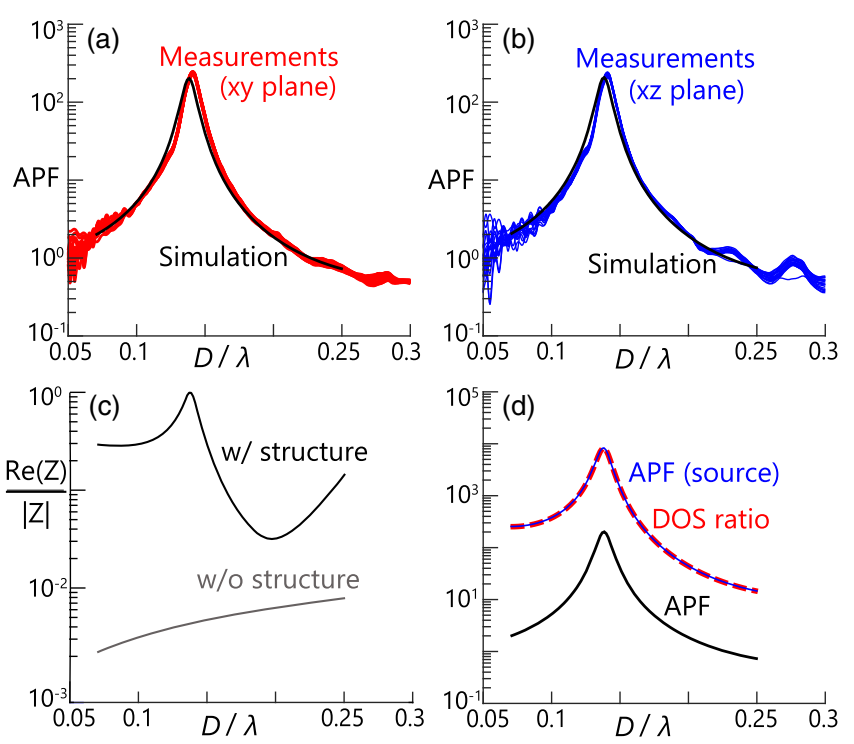

FIG. 4. Mechanisms of resonant enhancement of sound emission. (a)-(b) APF (= ratio of radiated power with and without the cavity) measured and simulated as a function of $D / \lambda$ for sound fields outside the structure $(r=15 \mathrm{~cm})$. (c) Ratio of the source's radiation resistance $\operatorname{Re}(Z)$, real part of impedance $Z$, to the module $|Z|$ is enhanced in the presence of the structure, indicating improved radiation efficiency by resonances. (d) Ratio of acoustic density of states (red dashed curve) coincides with the APF (blue curve) when calculated at the source surface, revealing the enhanced emission by the enhanced DOS; the black curve is a replot of (a) and (b) for comparison.

and per unit volume but in a sound system [26]. An analogy with quantum DOS follows because the steady-state Schrödinger equation in quantum mechanics takes the form of a Helmholtz equation. The solution to the equation can be written in terms of Green's function in complex form. The modes are picked up by the imaginary part of the Green's function $\operatorname{Im}\{G\}$. Then, the DOS (denoted by $\xi$ with the unit s/ $\mathrm{m}^{3}$ ) expressed in terms of $G(\cdot)$ is [26]

$$
\xi=-\frac{d k_{0}^{2}(\omega)}{d \omega} \frac{1}{\pi} \operatorname{Im}\left\{G\left(\omega, \vec{r}=\vec{r}_{0}, \vec{r}_{0}\right)\right\},
$$

where $\vec{r}$ is the detector location, $\vec{r}_{0}$ is the source location, and the wave dispersion relation of the medium is $k_{0}^{2}=$ $\omega^{2} / c_{0}^{2}$ for classical waves ( $c_{0}$ is the speed of sound) and $k_{0}^{2}=2 m \omega / \hbar$ for quantum $(\hbar$ is the reduced Planck constant and $m$ is particle mass).

We employed Eq. (1) to calculate the DOS of sound fields for both cases with (denoted by $\xi_{1}$ ) and without the enclosure (denoted by $\xi_{0}$ ). The Green's functions were obtained via the imaginary part of the simulated acoustic pressure at the source location where measurements are inaccessible. The resultant ratio $\xi_{1} / \xi_{0}$, shown in Fig. 4(d) (red dashed curve), is compared to the APF (black curve) that was previously shown in Figs. 4(a) and 4(b). They agree on the trend of dependence on $D / \lambda$, implying the 
enhanced emission via the acoustic DOS enhancement. The gap between the two curves is a result of losses of sound energy during propagation through the space-coiling structure, since the APF was calculated from integral of energy flux over a surface outside the structure, which is power radiated to far fields.

We evaluated the total emission power from the source by taking the integral of energy flux at the source surface for both cases with and without the structure. The resultant emission power enhancement, as shown by the blue curve in Fig. 4(d), matches perfectly with the DOS ratio (red dashed curve). In a macroscopic point of view, the enhanced DOS leads to enhanced power extinction from the source for a given source and frequency. This result complements to the aforementioned radiation efficiency that characterizes the energy radiated to the far field compared to the energy stored in the near field.

To explicitly show the perfect alignment of the increased DOS ratio with the enhanced emission power, we derived an analytical relation between the DOS in acoustics and emitted sound power. We started by assuming an acoustic monopole source located at $\vec{r}_{0}$ radiating monochromatic sound of angular frequency $\omega$ in air. Sommerfeld radiation boundary conditions were assumed. The wave equation with a source at $r_{0}$ is [25]

$$
\nabla^{2} p-\frac{1}{c_{\text {air }}^{2}} \frac{\partial^{2} p}{\partial t^{2}}=-\rho_{\text {air }} \frac{\partial Q(t)}{\partial t} \delta\left(\vec{r}-\vec{r}_{0}\right),
$$

where $\rho_{\text {air }}$ is the density of the air, $c_{\text {air }}$ is the speed of sound $c_{0}$ in the surrounding air, $p$ is the acoustic pressure, and $\delta$ is the Dirac delta function. The monopole source has a source strength (the volume flow rate from the source), $Q(t)=u_{s}(t) S_{s}$, where $u_{s}$ is the velocity on the surface of the source of a radius $a$ (and area $S_{s}=4 \pi a^{2}$ ). Assuming a temporal factor $e^{-i \omega t}$, we obtain

$$
\nabla^{2} p+\left(\omega^{2} / c_{\text {air }}^{2}\right) p=i \omega \rho_{\text {air }} u_{s} S_{s} \delta\left(\vec{r}-\vec{r}_{0}\right) .
$$

The corresponding Green's function of Eq. (3), under the same boundary condition, satisfies

$$
\nabla^{2} G\left(\omega, \vec{r}, \vec{r}_{0}\right)+k^{2} G\left(\omega, \vec{r}, \vec{r}_{0}\right)=\delta\left(\vec{r}-\vec{r}_{0}\right),
$$

where $k^{2}=\omega^{2} / c_{\text {air }}^{2}$. Combining Eqs. (3) and (4), we can express the acoustic pressure $p_{s}$ at the source location $\vec{r}_{0}$ in terms of $G\left(\omega, \vec{r}, \vec{r}_{0}\right)$ :

$$
p_{s}=i \omega \rho_{\mathrm{air}} u_{s} S_{s} G\left(\omega, \vec{r}=\vec{r}_{0}, \vec{r}_{0}\right) .
$$

The emission power of the sound source is calculated by the integral of time-averaged energy flux over the source surface, $P=0.5 \operatorname{Re}\left\{p_{s} u_{s}^{*}\right\} S_{s}$, where $u_{s}^{*}$ is the conjugate of $u_{s}$. Plugging Eq. (5) into $P$, we obtain the explicit relation between the power extinction from the source $P$ and the Green's function $G$ in a classical system:

$$
P=0.5 \omega \rho_{\text {air }} u_{s} S_{s} u_{s}^{*} S_{s} \operatorname{Re}\left\{i G\left(\omega, \vec{r}=\vec{r}_{0}, \vec{r}_{0}\right)\right\} .
$$

Comparing Eq. (6) with Eq. (1) for the DOS $\xi$, we obtain

$$
P=\pi \rho_{\text {air }} c_{\text {air }}^{2}|Q|^{2} \xi .
$$

which reveals the linear relation of the emitted power $P$ and the acoustic DOS $\xi$. Consequently, the acoustic Purcell effect has a consistent description by the enhanced power radiated to far fields (acoustic Purcell factor), the improved power extinction from the source, and the enhanced DOS $\xi$ of the sound system: $\mathrm{APF}=P_{1} / P_{0}=\xi_{1} / \xi_{0} \geq 1$.

Discussions. - The radiated power of a monopole source in free space is $P_{0}=|Q|^{2} k^{2} \rho_{\text {air }} c_{\text {air }} / 8 \pi$ [25]. We now rewrite Eq. (7) in terms of $P_{0}$. It becomes

$$
P=(2 \pi / \gamma) P_{0} \xi
$$

where $\gamma \equiv k^{2} / 4 \pi c_{\text {air }}$ is a source constant having the same dimension as the DOS $\xi$. Equation (8) is similar to the FGR in quantum mechanics that gives the emission rate of atoms in terms of density of states as [1,2],

$$
\Gamma=(2 \pi / \hbar)\left|\left\langle f\left|H^{\prime}\right| i\right\rangle\right|^{2} \xi_{q}
$$

where $\Gamma$ is the emission rate, $\xi_{q}$ the density of final states, and $\left\langle f\left|H^{\prime}\right| i\right\rangle$ the matrix element of the perturbing Hamiltonian $H^{\prime}$ between the initial state $i$ and the final state $f$. Classical waves show similar behavior, but on the level of field (not probability) amplitude: the emitted power $P$ of an acoustic source or the emission rate $\Gamma$ of a quantum source linearly depends on the acoustic DOS $\xi$ or the quantum one $\xi_{q}$, respectively. For the quantum FGR Eq. (9), the coefficients are $2 \pi / \hbar$ (constant term) and $\left|\left\langle f\left|H^{\prime}\right| i\right\rangle\right|^{2}$ (quadratic term); for the acoustic FGR Eq. (8), the coefficients are $2 \pi / \gamma$ (constant term) and the power $P_{0}$ (quadratic term).

Our formalism of acoustic analog to FGR can connect the radiation impedance $Z$ with the acoustic DOS $\xi$. Given the emitted power expressed as $P=\operatorname{Re}\{Z\}|Q|^{2} / 2 S_{s}$ [25], Eq. (8) leads to the linear dependence of $\operatorname{Re}\{Z\}$ on $\xi$ :

$$
\operatorname{Re}\{Z\}=(2 \pi / \gamma) R_{0} \xi,
$$

where $R_{0}=(k a)^{2} \rho_{\text {air }} c_{\text {air }}$ is the radiation resistance in free space. Consequently, our formalism has provided an alternative and complement to the conventional perspective that describes enhanced emission to the increased radiation resistance and resonances of the source-cavity system.

The acoustic analog to FGR that we have presented has served as the explicit foundation for describing the emission of acoustic sources and gives insight of how the emission is related to the density of states. The formalism makes it clear that the emission enhancement originates from the increase of the DOS and total power extinction 
from the source, which we have further validated via our experimental measurements and numerical simulations. The formalism could be applicable to examine sound transmission, reflection, absorption, and emission in general, and is not only limited to the emission enhancement. The enhanced emission is a phenomenon different from the enhanced transmission (see, for instance, Ref. [27]), where the power ratio in the presence to absence of the structure is at most one.

This work is supported by the start-up fund from the University of Mississippi. The Center for Manufacturing Excellence at the University of Mississippi is acknowledged for providing the 3D printing service. J. Z. and Y. W. acknowledge the support from King Abdullah University of Science and Technology.

M.L. and J.Z. contributed equally to this work. J.Z. proposed the theories, and M. L. conducted the experiments.

*Corresponding author.

zhang@olemiss.edu

† zhaojiajun1990@gmail.com

*ying.wu@kaust.edu.sa

[1] E. M. Purcell, Spontaneous emission probabilities at radio frequencies, Phys. Rev. 69, 681 (1946).

[2] P. Dirac, The quantum theory of the emission and absorption of radiation, Proc. Math. Phys. Eng. Sci. 114, 243 (1927).

[3] D. Kleppner, Inhibited Spontaneous Emission, Phys. Rev. Lett. 47, 233 (1981).

[4] P. Goy, J. Raimond, M. Gross, and S. Haroche, Observation of Cavity-Enhanced Single-Atom Spontaneous Emission, Phys. Rev. Lett. 50, 1903 (1983).

[5] E. Yablonovitch, Inhibited Spontaneous Emission in SolidState Physics and Electronics, Phys. Rev. Lett. 58, 2059 (1987).

[6] J. M. Gérard, B. Sermage, B. Gayral, B. Legrand, E. Costard, and V. Thierry-Mieg, Enhanced Spontaneous Emission by Quantum Boxes in a Monolithic Optical Microcavity, Phys. Rev. Lett. 81, 1110 (1998).

[7] H. N. Krishnamoorthy, Z. Jacob, E. Narimanov, I. Kretzschmar, and V. M. Menon, Topological transitions in metamaterials, Science 336, 205 (2012).

[8] M. A. Noginov, H. Li, Yu. A. Barnakov, D. Dryden, G. Nataraj, G. Zhu, C. E. Bonner, M. Mayy, Z. Jacob, and E. E. Narimanov, Controlling spontaneous emission with metamaterials, Opt. Lett. 35, 1863 (2010).

[9] Z. Jacob, I. I. Smolyaninov, and E. E. Narimanov, Broadband Purcell effect: Radiative decay engineering with metamaterials, Appl. Phys. Lett. 100, 181105 (2012).
[10] A. N. Poddubny, P. A. Belov, and Y. S. Kivshar, Purcell effect in wire metamaterials, Phys. Rev. B 87, 035136 (2013).

[11] A. Poddubny, I. Iorsh, P. Belov, and Y. Kivshar, Hyperbolic metamaterials, Nat. Photonics 7, 948 (2013).

[12] A. P. Slobozhanyuk, A. N. Poddubny, A. E. Krasnok, and P. A. Belov, Magnetic Purcell factor in wire metamaterials, Appl. Phys. Lett. 104, 161105 (2014).

[13] A. E. Krasnok, A. P. Slobozhanyuk, C. R. Simovski, S. A. Tretyakov, A. N. Poddubny, A. E. Miroshnichenko, Y. S. Kivshar, and P. A. Belov, An antenna model for the purcell effect, Sci. Rep. 5, 12956 (2015).

[14] Philip M. Morse and K. Uno Ingard, Theoretical Acoustics (Princeton University Press, Princeton, NJ, 1987).

[15] C. A Henricksen, Loudspeakers, Enclosures, and Headphones (Howard W. Sams Co., Indianapolis, IN, 1987).

[16] M. Fink, F. Lemoult, J. de Rosny, A. Tourin, and G. Lerosey, Subwavelength Focussing in Metamaterials Using Far Field Time Reversal, in Acoustic Metamaterials, edited by R. Craster and S. Guenneau (Springer, Dordrecht, 2013).

[17] K. Song, S. H. Lee, K. Kim, S. Hur, and J. Kim, Emission enhancement of sound emitters using an acoustic metamaterial cavity, Sci. Rep. 4, 4165 (2014).

[18] J. Zhao, L. Zhang, and Y. Wu, Enhancing monochromatic multipole emission by a subwavelength enclosure of degenerate mie resonances, J. Acoust. Soc. Am. 142, EL24 (2017).

[19] Z. Liang and J. Li, Extreme Acoustic Metamaterial by Coiling Up Space, Phys. Rev. Lett. 108, 114301 (2012).

[20] Yong Li, Bin Liang, Xu Tao, Xue-feng Zhu, Xin-ye Zou, and Jian-chun Cheng, Acoustic focusing by coiling up space, Appl. Phys. Lett. 101, 233508 (2012).

[21] Y. Cheng, C. Zhou, B. G. Yuan, D. J. Wu, Q. Wei, and X. J. Liu, Ultra-sparse metasurface for high reflection of lowfrequency sound based on artificial mie resonances, Nat. Mater. 14, 1013 (2015).

[22] X. Zhu, B. Liang, W. Kan, Y. Peng, and J. Cheng, DeepSubwavelength-Scale Directional Sensing Based on Highly Localized Dipolar Mie Resonances, Phys. Rev. Applied 5, 054015 (2016).

[23] X. Jiang, Y. Li, and L. Zhang, Thermoviscous effects on sound transmission through a metasurface of hybrid resonances, J. Acoust. Soc. Am. 141, EL363 (2017).

[24] The simulations in Ref. [18] were in 2D space.

[25] D. T. Blackstock, Fundamentals of Physical Acoustics (John Wiley \& Sons, New York, 2000).

[26] P. Sheng, Introduction to Wave Scattering, Localization and Mesoscopic Phenomena (Springer Science \& Business Media, 2006), Vol. 88.

[27] Y. Li, X. Jiang, B. Liang, J.-C. Cheng, and L. Zhang, Metascreen-Based Acoustic Passive Phased Array, Phys. Rev. Applied 4, 024003 (2015). 